\title{
GEOMETRIC REGULARIZATION ON RIEMANNIAN AND LORENTZIAN MANIFOLDS
}

\author{
SHANTANU DAVE, GÜNTHER HÖRMANN, AND MICHAEL KUNZINGER
}

\begin{abstract}
We investigate regularizations of distributional sections of vector bundles by means of nets of smooth sections that preserve the main regularity properties of the original distributions (singular support, wavefront set, Sobolev regularity). The underlying regularization mechanism is based on functional calculus of elliptic operators with finite speed of propagation with respect to a complete Riemannian metric. As an application we consider the interplay between the wave equation on a Lorentzian manifold and corresponding Riemannian regularizations, and under additional regularity assumptions we derive bounds on the rate of convergence of their commutator. We also show that the restriction to underlying space-like foliations behaves well with respect to these regularizations.
\end{abstract}

\section{INTRODUCTION}

We consider regularization processes to smooth out distributions on Riemannian and globally hyperbolic Lorentzian manifolds and investigate their compatibility with the wave-equation. The Riemannian setting has been addressed in [13] and we include an introduction to this approach. For a globally hyperbolic manifold we pick a splitting of the metric as obtained by 2 which provides us with a globally defined time function and a foliation by space-like hypersurfaces. The associated Riemannian metric naturally allows us to construct regularization processes on distributional sections of tensor-bundles and differential forms. We show that these regularizations interact nicely with the wave-equation on the Lorentzian manifold and with the foliation provided by the metric splitting.

By a regularization process we mean a net of smoothing operators that assigns a net of approximating smooth functions (or sections in a vector bundle) to any given distribution (or distributional section). We are interested in preserving a maximal set of regularity properties of the distribution in this process (support, singular support, wavefront set, Sobolev regularity). To assign such properties to approximating nets of smooth objects we employ the language of algebras of generalized functions in the sense of Colombeau ([9, 10, 31, 20]). In this approach any such regularization process provides an embedding of distributions into a space $\mathcal{G}(M)$ of nonlinear generalized functions (given by a quotient construction on spaces of approximating nets). This process preserves the regularity and singularity structure of the distributions as described in Section 2 below. The main interest in employing these regularizations is in studying non-smooth curved space-times.

1991 Mathematics Subject Classification. Primary 58J37; Secondary 46F30,46T30,35A27,53C50.

Key words and phrases. Regularization of distributions, complete Riemannian manifolds, globally hyperbolic Lorentz manifolds, algebras of generalized functions. 
Our approach is motivated by work on wave equations on non-smooth curved space-times. C.J.S. Clarke $([6,7,8])$ suggested to study physical fields for understanding the singularity structure of the space-time itself, i.e., to consider wave equations in low regularity. His work gave rise to a number of further studies. In particular, in the framework of generalized functions this line of research was pursued in [34, 19, 33]. For a more detailed introduction we refer to [25].

Thus let us consider a model situation and analyze the generalized solutions to wave equations corresponding to a generalized Lorentz metric $\tilde{g}$ on the smooth manifold $M$ (cf. 20, 27, 19]), i.e., a global Cauchy problem with initial data $a, b \in$ $\mathcal{D}^{\prime}(S)$ on a suitable initial value surface $S$ in the form

$$
\square_{\tilde{g}} u=0,\left.\quad u\right|_{S}=\iota(a),\left.\nabla_{\mathrm{n}} u\right|_{S}=\iota(b),
$$

where $\iota$ is assumed to be an embedding of distributions into the algebra of generalized functions.

Suppose that the generalized Lorentzian structure induced by $\tilde{g}$ allows a splitting of the wave operator in the form $\square_{\tilde{g}}=\gamma \cdot\left(\square_{g}+Q\right)$, where $g$ is a smooth "background" metric, $\gamma$ is a positive measure or a strictly positive generalized function, and $Q$ is a partial differential operator with distributions or generalized functions as coefficients, but whose coefficient singularities are concentrated in certain spacetime regions. A simple example is a generalized Robertson-Walker space-time with $\tilde{g}=-d t^{2}+(1+\mu(t)) h$ on $M=\mathbb{R} \times S$, where $h$ is a smooth Riemannian metric on the smooth manifold $S$ and $\mu$ is a nonnegative generalized function. In this case $g=-d t^{2}+h$ can serve as a smooth background metric and the above splitting would involve $\gamma=1 /(1+\mu)$ and $Q=Q\left(t, \partial_{t}\right)$, which acts only on the one-dimensional factor.

If we impose the splitting assumption as above, then we have for any generalized function $u \in \mathcal{G}(M)$ that the equation $\square_{\tilde{g}} u=0$ is equivalent to $\square_{g} u+Q u=0$. Let $u \in \mathcal{G}(M)$ be a solution to the Cauchy problem stated above. In attempting to extract its distributional aspects or assess its singularity structure we could adopt the following strategy. Let $v$ be the distributional solution (assuming that it exists and is unique) to

$$
\square_{g} v=0,\left.\quad v\right|_{S}=a,\left.\quad \nabla_{\mathrm{n}} v\right|_{S}=b,
$$

and put $w:=\iota(v)-u \in \mathcal{G}(M)$ as a comparison of the generalized function $u$ with its "background distributional aspect". Now, if the embedding ८ commutes with restriction to $S$ and with $\square_{g}$, then simple manipulations allow us to draw the conclusion that $w$ satisfies the following Cauchy problem:

$$
\square_{g} w=Q u,\left.\quad w\right|_{S}=0,\left.\quad \nabla_{\mathrm{n}} w\right|_{S}=0 .
$$

Thus, $w$ satisfies an inhomogeneous Cauchy problem corresponding to the smooth background metric $g$ and with generalized functions occurring only on the righthand side of the equation. In particular, $w$ will be smooth in regions where null geodesics corresponding to $g$ emanating from $S$ do not intersect the $\mathcal{C}^{\infty}$-singular support of $Q u$.

In the more general situation of a globally hyperbolic Lorentz manifold provided with a foliation into spacelike hypersurfaces via a splitting of the metric we shall construct an embedding $\iota$ and show that the embedding almost commutes with $\square_{g}$ and with restriction to space-like slices of the foliation. The extent of failure to commute is roughly measured by the divergence of the volume element on the 
slices along the time direction. Although we state our results in the scalar case, the extension to the case of wave-equations on differential forms is obvious.

\section{Regularization of Distributions on COMPlete RiEmannian ManifoldS}

In this section we describe a geometric regularization procedure for distributions (or distributional sections of vector bundles) on complete Riemannian manifolds. This procedure encodes regularity and singularity features in terms of asymptotic behavior. Our approach is based on [13, to which we refer for further details.

We first fix some notations from the theory of distributions. Let $M$ be an orientable complete Riemannian manifold of dimension $n$ with Riemannian metric $g$. The space $\mathcal{D}^{\prime}(M)$ of Schwartz distributions on $M$ is the dual of the space $\Omega_{c}^{n}(M)$ of compactly supported $n$-forms on $M$. Further, $\mathcal{D}(M)$ is the space of smooth compactly supported functions on $M$. We identify $\mathcal{D}(M)$ with $\Omega_{c}^{n}(M)$ via $f \mapsto f \cdot d g$, with $d g$ the Riemannian volume form induced by $g$. Thus $\mathcal{D}^{\prime}(M)$ can be viewed as the dual space of $\mathcal{D}(M)$. We consider $L_{\text {loc }}^{1}(M)$ (hence in particular $\left.\mathcal{C}^{\infty}(M)\right)$ a subspace of $M$ via $f \mapsto\left[\varphi \mapsto \int_{M} f \varphi d g\right]$. If $E$ is a vector bundle over $M$ then $\mathcal{D}^{\prime}(M: E)$, the space of $E$-valued distributions on $M$ is defined by $\mathcal{D}^{\prime}(M$ : $E)=\mathcal{D}^{\prime}(M) \otimes_{\mathcal{C}^{\infty}(M)} \Gamma^{\infty}(M: E)$, with $\Gamma^{\infty}(M: E)$ the space of smooth sections of $E$. By $\mathcal{E}^{\prime}(M: E)$ we denote the space of compactly supported distributional sections of $E$. We shall assume that $E$ is endowed with a Hermitian inner product so that the distributional sections $\mathcal{D}^{\prime}(M: E)$ of $E$ can be identified with the dual $\Gamma_{c}^{\infty}(M: E)^{\prime}$ of the space of compactly supported smooth sections. Regularity properties of distributions we are interested in are, in particular, encoded in the singular support $\operatorname{singsupp}(w)$, the wavefront set $\mathrm{WF}(w)$, and the Sobolev regularity of any given $w \in \mathcal{D}^{\prime}(M)$.

In order to be able to track these regularity properties in terms of regularizations of a given distribution we need a conceptual framework that allows to assign geometrical and analytical properties to regularizations, i.e., to nets of smooth functions. Algebras of generalized functions in the sense of J. F. Colombeau ([9, 10, 31]) indeed provide a well-developed theory of this kind and below we shall briefly review some basic definitions, based mainly on [20, 16.

The basic idea of Colombeau's approach is to assign to any given locally convex space $X$ a space $\mathcal{G}_{X}$ of generalized functions as follows. We define the space $\mathcal{M}_{X}$ of moderate nets to consist of those maps $\varepsilon \mapsto x_{\varepsilon}(\varepsilon \in I:=(0,1])$ that are smooth and for any seminorm $\rho$ of $X$ satisfy $\rho\left(X_{\varepsilon}\right)=O\left(\varepsilon^{N}\right)$ for some integer $N$ as $\varepsilon \rightarrow 0$. Similarly we call a net $x_{\varepsilon}$ negligible if for all seminorms $\rho$ and all integers $N$ the asymptotic relation $\rho\left(X_{\varepsilon}\right) \sim O\left(\varepsilon^{N}\right)$ holds. The space $\mathcal{G}_{X}$ is defined as the quotient of the space of all moderate nets by the space $\mathcal{N}_{X}$ of all negligible nets. The class represented by a net $x_{\varepsilon}$ shall be denoted by $\left[x_{\varepsilon}\right]$. In case $M$ is a smooth manifold we call $\mathcal{G}(M):=\mathcal{G}_{\mathcal{C} \infty(M)}$ the standard (special) Colombeau algebra of generalized functions on $M([9,12,20])$. If $E \rightarrow M$ is a vector bundle then we set $\mathcal{G}(M: E):=\mathcal{G}_{\Gamma^{\infty}(M: E)}$. For $E=\mathbb{C}$ the space $\mathcal{G}_{\mathbb{C}}$ inherits a ring structure from $\mathbb{C}$. It is therefore called the space of generalized numbers, denoted by $\tilde{\mathbb{C}}$. Every space $\mathcal{G}_{E}$ is naturally a $\tilde{\mathbb{C}}$-module, and hence is called the $\widetilde{\mathbb{C}}$-module associated with $E$ ([16]).

Similarly we shall also consider the subspace $\mathcal{G}_{X}^{\infty}$ of regular elements of $\mathcal{G}_{X}$, defined as the space of all those elements of $\mathcal{G}_{X}$ that can be represented by a net $x_{\varepsilon}$ for which one can find an integer $N$ in the above relations independent of any 
seminorm $\rho$ on $X$. Thus $\mathcal{G}_{X}^{\infty}$ is the space of uniformly controlled asymptotics. Again we set $\mathcal{G}^{\infty}(M):=\mathcal{G}_{\mathcal{C}^{\infty}(M)}^{\infty}$. In regularity theory, $\mathcal{G}^{\infty}(M)$ is the analogue of $\mathcal{C}^{\infty}(M)$ in the theory of distributions ([31, 24, 14, 17]). This is based on the fundamental result ([31]) that for open subsets $\Omega$ of $\mathbb{R}^{n}, \iota\left(\mathcal{D}^{\prime}(\Omega)\right) \cap \mathcal{G}^{\infty}(\Omega)=\mathcal{C}^{\infty}(\Omega)$, where $\iota$ is the standard embedding of $\mathcal{D}^{\prime}$ into $\mathcal{G}$ via convolution with a standard mollifier. We will introduce further notions of regularity theory based on $\mathcal{G}^{\infty}$ below.

The assignment $X \mapsto \mathcal{G}_{X}$ is obviously functorial, so that any continuous linear map $\phi: X \rightarrow Y$ naturally induces a map $\phi_{*}: \mathcal{G}_{X} \rightarrow \mathcal{G}_{Y}$. Thus a smooth map $f: M \rightarrow N$ gives rise to a pull-back $f^{*}: \mathcal{G}(M) \rightarrow \mathcal{G}(M)$. In particular $\mathcal{G}(M)$ defines a fine sheaf of algebras and similarly $\mathcal{G}(M: E)$ is a fine sheaf of $\mathcal{G}(M)$ modules.

We finally introduce the notion of association, which provides a concept of weak equality between elements of Colombeau spaces as well as a standardized way of assigning distributional limits to certain Colombeau generalized functions. For $u$, $v \in \mathcal{G}(M: E)$ we say that $u$ is associated (or weakly equal) to $v, u \approx v$ if $u_{\varepsilon}-v_{\varepsilon} \rightarrow 0$ in $\mathcal{D}^{\prime}(M: E)$ for (some, hence any) representatives of $u, v$. We say that $u$ possesses $w \in \mathcal{D}^{\prime}(M: E)$ as a distributional shadow if $u_{\varepsilon} \rightarrow w$ in $\mathcal{D}^{\prime}(M: E)$.

To construct the desired regularization process on $\mathcal{D}^{\prime}(M: E)$ we need two pieces of data:

(1) A Schwartz function $F \in \mathscr{S}(\mathbb{R})$ such that $F \equiv 1$ near the origin.

(2) An elliptic symmetric differential operator $D$ on $E$ such that the speed of propagation $C_{D}$, defined as

$$
C_{D}=\sup \left\{\left\|\sigma_{D}(x, \xi)\right\| \mid x \in M,\|\xi\|=1\right\}
$$

is finite. Here $\sigma_{D}$ denotes the principal symbol of $D$. Such an operator $D$ will be referred to as admissible.

As a consequence of the finite speed of propagation, the symmetric operator $D$ is essentially self-adjoint ([23, 10.2.11]). Therefore the equation

$$
\frac{\partial}{\partial t} u=i D u \quad u(., 0)=u_{0},
$$

has a unique solution for all times $t$ for any initial datum $u_{0} \in \Gamma_{c}^{\infty}(M: E)$. Indeed it follows from functional calculus that $e^{i t D} u_{0}$ is a solution, and uniqueness can be established using energy estimates.

We note that functional calculus defines a map

$$
\mathscr{S}(\mathbb{R}) \ni f \mapsto f(D) \in \mathcal{B}\left(L^{2}(M: E)\right)
$$

where the operator $f(D)$ can also be expressed by the Fourier inversion formula:

$$
f(D):=\frac{1}{2 \pi} \int_{\mathbb{R}} \hat{f}(s) e^{i s D} d s
$$

(in the sense of strong operator convergence). Since the operator $D$ is elliptic, it follows that $f(D)$ has a smooth kernel by elliptic regularity.

Let $F \in \mathscr{S}(\mathbb{R})$ with $F \equiv 1$ near the origin and set $F_{\varepsilon}(x):=F(\varepsilon x)$. Then we shall obtain the desired regularizing procedure from $F_{\varepsilon}(D)$. To do this we first take a closer look at singularity properties available in generalized functions.

As has been mentioned above the space $\mathcal{G}(M: E)$ is a fine sheaf over $M$, hence provides a notion of support. Furthermore, the notion of wave-front set can be defined for generalized functions both locally and in invariant global terms analogous 
to distributions. For $\Omega \subseteq \mathbb{R}^{n}$ open we call $u \in \mathcal{G}(\Omega) \mathcal{G}^{\infty}$-microlocally regular at $\left(x_{0}, \xi_{0}\right) \in T^{*} \Omega \backslash 0$ if there exists some test function $\varphi \in \mathcal{D}(\Omega)$ with $\varphi\left(x_{0}\right)=1$ and a conic neighborhood $\Gamma \subseteq \mathbb{R}^{n} \backslash 0$ of $\xi_{0}$ such that the Fourier transform $\mathcal{F}(\varphi u)$ is rapidly decreasing in $\Gamma$, i.e., there exists $N$ such that for all $l$,

$$
\sup _{\xi \in \Gamma}(1+|\xi|)^{l}\left|\left(\varphi u_{\varepsilon}\right)^{\wedge}(\xi)\right|=O\left(\varepsilon^{-N}\right) \quad(\varepsilon \rightarrow 0) .
$$

The generalized wave front set of $u, \mathrm{WF}_{g}(u)$, is the complement of the set of points $\left(x_{0}, \xi_{0}\right)$ where $u$ is $\mathcal{G}^{\infty}$-microlocally regular. By [21], for any $u \in \mathcal{G}(M), \mathrm{WF}_{g}(u)$ can naturally be viewed as a subset of $T^{*} M \backslash 0$. It is equivalently defined as (cf.[17]),

$$
\mathrm{WF}_{g}(u)=\bigcap_{P u \in \mathcal{G}^{\infty}(M)} \operatorname{char}(P) \quad\left(P \in \Psi_{c l}^{0}(M)\right) .
$$

Here $\operatorname{char}(P) \subseteq T^{*} M$ is the characteristic set of the order 0 classical pseudodifferential operator $P$. The singular support of $u, \operatorname{singsupp}_{g}(u)$, is the complement of the maximal open set on which $u \in \mathcal{G}^{\infty}$. It then follows that $\operatorname{singsupp}_{g}(u)=\operatorname{pr}_{1}\left(\mathrm{WF}_{g}\right)$.

We are now ready to define a class of regularization procedures for distributional sections of a vector bundle.

\subsection{Regularizations.}

Definition 2.1. A parametrized family $\left(T_{\varepsilon}\right)_{\varepsilon \in I}$ of properly supported smoothing operators (in the sense of [4, ch. 1.4]) is called an optimal regularization process if

(1) The regularization of any compactly supported distributional section $s \in$ $\mathcal{E}^{\prime}(M: E)$ is of moderate growth: For any continuous seminorm $\rho$ on $\Gamma^{\infty}(M: E)$, there exists some integer $N$ such that

$$
\rho\left(T_{\varepsilon} s\right)=O\left(\varepsilon^{N}\right) \quad(\varepsilon \rightarrow 0) .
$$

(2) The net $\left(T_{\varepsilon}\right)$ is an approximate identity: for each $s \in \mathscr{E}^{\prime}(M: E)$,

$$
\lim _{\varepsilon \rightarrow 0} T_{\varepsilon} s=s \quad \text { in } \mathcal{D}^{\prime}(M: E) .
$$

(3) If $u \in \Gamma_{c}^{\infty}(M: E)$ is a smooth compactly supported section of $E$ then for all continuous seminorms $\rho$ and given any integer $m$,

$$
\rho\left(T_{\varepsilon} u-u\right)=O\left(\varepsilon^{m}\right) .
$$

(4) The induced map $\iota_{T}: \mathcal{E}^{\prime}(M: E) \rightarrow \mathcal{G}(M: E)$ preserves support, and extends to a sheaf map $\mathcal{D}^{\prime}(M: E) \rightarrow \mathcal{G}(M: E)$ that satisfies,

$$
\iota_{T}\left(\mathcal{D}^{\prime}(M: E)\right) \cap \mathcal{G}^{\infty}(M: E)=\Gamma^{\infty}(M: E) .
$$

This implies in particular that $\iota_{T}$ preserves singular support.

(5) The map $\iota_{T}$ preserves wave-front sets in the sense that for any distribution $s \in \mathcal{D}^{\prime}(M: E)$

$$
\mathrm{WF}(u)=\mathrm{WF}_{g}\left(\iota_{T}(u)\right) .
$$

As mentioned already we shall use spectral properties of the elliptic differential operators to obtain regularizing processes satisfying the above conditions.

Our main result in this section is the following.

Theorem 2.2. Let $F \in \mathscr{S}(\mathbb{R})$ be a Schwartz function such that $F \equiv 1$ near the origin. Let $F_{\varepsilon}(x):=F(\varepsilon x)$. Given an admissible differential operator D, the family of operators $\left(F_{\varepsilon}(D)\right)_{\varepsilon \in I}$ provides an optimal regularization process in sense of Def. 2.1. 
The essential idea of the proof boils down to the following two steps.

First we prove Theorem 2.2 under the assumption that the underlying manifold $M$ is compact. We then use finite propagation speed to extend the result to a general complete Riemannian manifold.

Thus let us for the time being assume that $M$ is a closed manifold. Then the space of smoothing operators $\Psi^{-\infty}(M: E)$ is a Frechét algebra. The functional calculus map defined by $D$ induces a smooth map $\phi_{D}: \mathscr{S}(\mathbb{R}) \rightarrow \Psi^{-\infty}(M: E)$, hence the estimate 1 holds because $F_{\varepsilon}$ is a moderate net and $F_{\varepsilon}(D)=\left(\phi_{D}\right)_{*}\left(F_{\varepsilon}\right)$, where $\left(\phi_{D}\right)_{*}$ is the induced map on the asymptotic spaces $\mathcal{G}_{\mathscr{S}(\mathbb{R})} \rightarrow \mathcal{G}_{\Psi^{-\infty}(M)}$. Condition 2 then follows as the ring map $\phi_{D}$ preserves approximate units.

When $M$ is compact the operator $D$ has discrete spectrum and the spectrum of $D^{2}$ satisfies Weyl's law, namely

$$
N_{D^{2}}(\lambda):=\#\left\{\lambda_{i} \in \operatorname{sp}\left(D^{2}\right) \mid \lambda_{i} \leq \lambda\right\} \sim C \lambda^{\frac{\operatorname{dim}(M)}{2}} .
$$

The Weyl estimates in conjunction with the fact that $F \equiv 1$ near the origin now provides the compatibility condition 3] (see [11]).

To describe the regularity of a distributional section $u \in \mathcal{D}^{\prime}(M: E)$ we first note that any distribution provides a map between two Frechét spaces, namely between the smoothing operators $\Psi^{-\infty}(M: E)$ and the smooth sections $\Gamma^{\infty}(M: E)$ by evaluation. More precisely, to any $u \in \mathcal{D}^{\prime}(M: E)$ we associate the map

$$
\Theta_{u}: \Psi^{-\infty}(M) \rightarrow \Gamma^{\infty}(M: E) \quad \Theta_{u}(T):=T(u) .
$$

The mapping properties of the maps $\Theta_{u}$ imply that if $u \notin H^{k}(M: E)$ for every $k>t$ then given any $\delta>0,\left\|F_{\varepsilon}(D) u\right\|_{L^{2}(M: E)}^{2}$ is not $O\left(\varepsilon^{\frac{\operatorname{dim} \mathrm{M}}{2}+t+\delta}\right)$ (see [11, Lemma 7.4]). This direct description of Sobolev regularity is in fact stronger than condition 4 and therefore,

$$
\left.\iota_{F_{\varepsilon}(D)}\left(\mathcal{D}^{\prime}(M: E)\right) \cap \mathcal{G}^{\infty}(M: E)=\Gamma^{\infty}(M: E)\right) .
$$

The sheaf property of the embedding $\iota_{F_{\varepsilon}(D)}$ as well as the proof of the result for the more general case of a not necessarily compact complete manifold rely on the finite speed of propagation of $D$, to which we turn next.

2.2. Finite speed of propagation. We shall reduce the computation of asymptotics on a complete Riemannian manifold to certain compact manifolds obtained as doubles of suitable compact submanifolds with boundaries. If $X$ is a compact manifold with boundary, a double of $X$, denoted here by $D X$ is a closed manifold obtained by gluing two copies of $X$ along the boundary $\partial X$ (see, e.g., [26]). If $X$ is a compact manifold with boundary embedded in a Riemannian manifold $M$ of the same dimension and if $U$ is an open subset of $M$ such that $\bar{U} \subset$ interior $(X)$, then there exists a Riemannian metric on $D X$ such that the inclusion $j: U \hookrightarrow D X$ becomes an isometric embedding. Moreover, for any vector bundle $E \rightarrow M$ there exists a corresponding vector bundle $E_{X} \rightarrow D X$ such that $\left.E_{X}\right|_{U}$ is canonically isomorphic to $E_{\left.\right|_{U}}$. Also, there exists a symmetric elliptic operator $D_{X}$ on $E_{X}$ that coincides with $D$ on $U$.

Let $u \in \mathcal{E}^{\prime}(M: E)$ and fix a constant $c>0$. Then by the Hopf Rinow theorem the open ball $U:=B_{2 c \cdot C_{D}}\left(\operatorname{supp}(u)\right.$ ) (with $C_{D}$ as defined in (2.1)) is relatively compact and therefore contained in a compact manifold with boundary $X \subseteq M$. By the above $u$ can be identified with a distributional section of $E_{X} \rightarrow D X$. 
Proposition 2.3. With assumptions on $u, c$ and $F$ as above $F(D) u$ and $F\left(D_{X}\right) u$ are both supported in $U$ and

$$
F(D) u=F\left(D_{X}\right) u .
$$

Proof. The restrictions of $D$ and $D_{X}$ to the open set $U$ coincide, hence uniqueness of solutions to (2.2) implies that $e^{i s D} u$ and $e^{i s D_{X}} u$ agree for $s \leq c$. Hence the claim follows from the Fourier Inversion Formula (2.3).

From these observations the proof of Th. 2.2 can be deduced along the following lines (cf. [13, Sec. 4]):

Let $\phi \in \mathcal{D}(-c, c)$ satisfy $\phi \equiv 1$ in a neighborhood of 0 and let $u \in \mathcal{E}^{\prime}(M: E)$. Then

$$
F_{\varepsilon}(D) u=\frac{1}{2 \pi} \int_{-\infty}^{\infty} \phi(s) \hat{F}_{\varepsilon}(s) e^{i s D} u d s=j^{*}\left(\frac{1}{2 \pi} \int_{-\infty}^{\infty} \phi(s) \hat{F}_{\varepsilon}(s) e^{i s D_{X}} u d s\right)
$$

Based on this calculation we observe that the estimates required for Definition 2.1 in the general case of $F_{\varepsilon}(D)$ follow from that of $F_{\varepsilon}\left(D_{X}\right)$ which have already been established in case of the closed manifold $D X$. Furthermore the sheaf properties of $\iota_{F_{\varepsilon}(D)}$ can be obtained as a consequence of the calculations in Proposition 2.3 The support of $u$ coincides with the generalized support of $\left[F_{\varepsilon}(D) u\right]$. This implies that the embedding extends to a sheaf morphism $i_{F_{\varepsilon}}: \mathcal{D}^{\prime}(M: E) \rightarrow \mathcal{G}(M: E)$. Preservation of wavefront sets is more involved. We refer to [13, Th. 3.10] for a complete proof of this property.

Remark 2.4. In the scalar case, an alternative proof (not relying on the above doubling-technique) of Theorem 2.2 can be found in [13], Section 3.

2.3. Applications. As a first application consider $E=\wedge^{*} M$, the exterior bundle over a Riemannian manifold $M$ and $D=d+d^{*}$ where $d^{*}$ is the Hodge adjoint of the de Rham differential $d$. Then $D$ is symmetric, elliptic and has propagation speed $C_{D}=1$ since $\sigma_{D}(x, \xi)^{2}=-\|\xi\|^{2} \mathrm{id}$, hence is essentially self-adjoint. Letting $\Delta:=D^{2}$ be the Laplace-Beltrami operator on $\Omega^{*}(M)$, it follows from functional calculus that $\cos (s D)=\cos (s \sqrt{\Delta})$ on $L^{2}(M)$.

Now let $F$ be an even Schwartz function which has germ 1 at the origin. Then

$$
F(\sqrt{\Delta})=\frac{1}{2 \pi} \int_{-\infty}^{\infty} \hat{F}(s) \cos (s \sqrt{\Delta}) d s
$$

as a Bochner integral in $\mathcal{B}\left(L^{2}(M)\right)$.

Finally, let $c>0$ and pick an even test function $\phi_{c}$ with support in $(-2 c, 2 c)$ and such that $\phi_{c} \equiv 1$ on $(-c, c)$. Now set

$$
T_{\varepsilon}(\sqrt{\Delta}):=\frac{1}{2 \pi} \int_{-\infty}^{\infty} \phi_{c}(s)\left(F_{\varepsilon}\right)^{\wedge}(s) \cos (s \sqrt{\Delta}) d s .
$$

Then each $T_{\varepsilon}(\sqrt{\Delta})$ is a properly supported smoothing operator and $\left(T_{\varepsilon}(\sqrt{\Delta})\right)_{\varepsilon \in I}$ is an optimal regularization process in the sense of Def. 2.1 From the construction of $T$ in terms of the functional calculus of the Laplace operator we conclude the following invariance properties of the corresponding embedding $\iota_{T}$ :

\section{Corollary 2.5.}

(i) Let $f: M \rightarrow M$ be an isometry. Then for any $u \in \mathcal{D}^{\prime}(M), \iota_{T}\left(f^{*} u\right)=$ $f^{*} \iota_{T}(u)$. 
(ii) If $\Psi$ is a pseudodifferential operator commuting with $\Delta$, then $\Psi$ commutes with $\iota_{T}$.

For the special case $\mathbb{R}^{n}$ with the Euclidean metric the above construction gives

$$
T_{\varepsilon} u=\mu_{\varepsilon} * u, \quad \text { with } \quad \widehat{\mu_{\varepsilon}}(\xi)=\frac{1}{2 \pi} \mathcal{F}\left(\phi \frac{1}{\varepsilon} \widehat{F}(\dot{\bar{\varepsilon}})\right)(|\xi|) .
$$

In particular, for the one-dimensional case $n=1$ we obtain $\mu_{\varepsilon}=\phi_{c} \cdot \widehat{F}(. / \varepsilon) /(2 \pi \varepsilon)$. Note that $\widehat{F}(. / \varepsilon) /(2 \pi \varepsilon)$ is a standard mollifier, i.e., a Schwartz function with unit integral and all higher moments vanishing. Thus the regularization process reduces precisely to the usual Colombeau embedding via convolution ([10, 31, 20]).

A further consequence of the above construction is that it relates naturally to isomorphisms of vector bundles. Thus let $\phi: E_{1} \rightarrow E_{2}$ be a vector-bundle isomorphism (covering the identity map on $M$ ) and let $D_{1}$ be an order one admissible operator on $E_{1}$. We choose a Hermitian structure on $E_{2}$ which makes $\phi$ an isometry. Then the push-forward operator $D_{2}:=\phi D_{1} \phi^{-1}$ is isospectral to $D_{1}$. The naturality of functional calculus then gives that for any Schwartz function $F$ with $F \equiv 1$ near the origin the embedding and the bundle map $\phi$ commute, that is

$$
\phi \circ F_{\varepsilon}\left(D_{1}\right)=F_{\varepsilon}\left(D_{2}\right) \circ \phi .
$$

We note that if $r_{1}+s_{1}=r_{2}+s_{2}$ then any Riemannian metric provides an isomorphism of the tensor bundles $\mathfrak{T}_{s_{1}}^{r_{1}} \rightarrow \mathfrak{T}_{s_{2}}^{r_{2}}$ by 'raising or lowering of indices'. Thus if we pick the connection Laplace operators on the tensor bundles and an even Schwartz function $F$ as above we obtain a regularization process that is well behaved with respect to raising and lowering of indices.

\section{Regularization on GLOBALly hyPERBOLIC SPACE-TIMES}

We now return to the situation described at the end of Section 1 . Thus, throughout this section, $(M, g)$ will be a smooth space-time, i.e., a connected time-oriented Lorentz manifold. We first review the concept of metric splitting for globally hyperbolic space-times. Building on this we construct invariant regularizations of distributions on smooth globally hyperbolic space-times.

3.1. The metric splitting of globally hyperbolic space-times and associated Riemann metrics. The original definition, due to J. Leray of global hyperbolicity of a space-time $M$ appeared in 28. It requires that the set of causal curves connecting two points $p, q \in M$ be compact in the space of all rectifiable paths with respect to a suitable metric topology ([5, Ch. XII, Sec. 8, 9]). In what follows, we will use an equivalent definition, cf. [22, Sec. 6.6], and [5, Ch. XII, Th. 10.2].

Thus we call a space-time globally hyperbolic if it satisfies (a) strong causality and (b) for any $p, q \in M$ the intersection $J^{+}(p) \cap J^{-}(q)$ of the causal future $J^{+}(p)$ of $p$ with the causal past $J^{-}(q)$ of $q$ is a compact subset of $M$. Thanks to [3] condition (a) may be weakened to causality, i.e., non-existence of closed time-like curves.

As shown by Geroch (cf. [18]) global hyperbolicity is equivalent to the existence of a Cauchy hypersurface and in turn provides a foliation of $M$ by Cauchy hypersurfaces. Further development of these constructions and techniques led to the following result due to Bernal-Sánchez (cf. [2]) on the so-called metric splitting of a globally hyperbolic space-time $(M, g)$ : There exists a Cauchy hypersurface $S$ in 
$M$ and an isometry of $(M, g)$ with the Lorentz manifold $(\mathbb{R} \times S, \lambda)$ with Lorentz metric $\lambda$ given by

$$
\lambda=-\beta d t^{2}+h_{t},
$$

where $\beta \in \mathcal{C}^{\infty}(\mathbb{R} \times S)$ is positive, $\left(h_{t}\right)_{t \in \mathbb{R}}$ is a smoothly parametrized family of Riemannian metrics on $S$, and $t$ denotes (slightly ambiguously) both the global time function $(t, x) \mapsto t$ and its values. In other words, in order to construct a regularization (or embedding) for distributions on the globally hyperbolic Lorentzian manifold $(M, g)$ we may as well assume that $(M, g)=(\mathbb{R} \times S, \lambda)$.

The specific structure of the Lorentz metric $\lambda$ in (3.1) suggests to associate with it the Riemann metric

$$
\rho:=\beta d t^{2}+h_{t}
$$

on $\mathbb{R} \times S$ and to simply use the regularization and embedding of distributions on $\mathbb{R} \times$ $S$ based on this Riemann metric $\rho$. The regularization construction on Riemannian manifolds described above requires completeness of the Riemann metric $\rho$. It may happen that $\rho$ is not complete, however we may then introduce an appropriate conformal factor to obtain a complete Riemannian metric (cf. [30]). Note that the latter would amount to introducing the exact same conformal factor for the Lorentz metric $\lambda$ and would not change the class in the so-called causal hierarchy of spacetimes according to Minguzzi-Sánchez [29]. Therefore we assume henceforth that $\rho$ is complete.

Denoting by $\Delta_{\rho}$ the Laplacian w.r.t. $\rho$, for any $s \in \mathbb{R}$ and any $u \in \mathcal{D}(M)$ we set

$$
\|u\|_{s}:=\left\|\left(1+\Delta_{\rho}\right)^{s / 2} u\right\|_{L^{2}(M)} .
$$

The Sobolev space $H^{s}(M)$ of order $s$ is the completion of $\mathcal{D}(M)$ with respect to this norm.

Example. (i) Consider $S=\mathbb{R}$ and $\lambda=-d t^{2}+\frac{d x^{2}}{1+t^{6} x^{6}}$ as Lorentz metric on $\mathbb{R}^{2}$. It is elementary to check that $\left(\mathbb{R}^{2}, \lambda\right)$ is globally hyperbolic, e.g., by showing that $\{0\} \times \mathbb{R}$ is a Cauchy hypersurface. The associated Riemann metric $\rho=d t^{2}+\frac{d x^{2}}{1+t^{6} x^{6}}$ is not complete, since the hypersurfaces $\{t\} \times \mathbb{R}$ with $t \neq 0$ are closed and bounded but not compact in $\left(\mathbb{R}^{2}, \rho\right)$. Multiplying $\rho$ by the function $\alpha \in \mathcal{C}^{\infty}\left(\mathbb{R}^{2}\right), \alpha(t, x)=1+t^{6} x^{6}$, yields a conformal metric which is complete (by the Hopf-Rinow theorem, since lengths of curves w.r.t. $\alpha \cdot \rho$ are greater than or equal to their Euclidean lengths).

(ii) Let $\left(S, h_{0}\right)$ be a connected Riemannian manifold. For the Robertson-Walker space-time $\mathbb{R} \times S$ with Lorentz metric of the form $\lambda=-d t^{2}+f(t)^{2} h_{0}$, where $f \in \mathcal{C}^{\infty}(\mathbb{R})$ is positive, we have (cf. [1, Lemma A.5.14]):

$(\mathbb{R} \times S, \lambda)$ is globally hyperbolic if and only if $\left(S, h_{0}\right)$ is complete.

If this is the case, then the corresponding Riemannian metric $\rho=d t^{2}+f(t)^{2} h_{0}$ on $\mathbb{R} \times S$ is complete (see 32, Lemma 7.40).

3.2. Regularization and embedding via the associated Riemannian structure. Let $\left(T_{\varepsilon}\right)_{\varepsilon \in I}$ be the regularization (i.e., family of properly supported smoothing operators) and $\iota: \mathcal{D}^{\prime}(\mathbb{R} \times S) \hookrightarrow \mathcal{G}(\mathbb{R} \times S)$ be the embedding associated with the complete Riemann metric $\rho$ as in (2.6). We recall that the embedding thus respects the differential algebraic structure of $\mathcal{C}^{\infty}(\mathbb{R} \times S)$ and the wave front sets of distributions in the strong sense of generalized functions, that is, with respect to equality on the level of $\mathcal{G}(\mathbb{R} \times S)$. Furthermore, $\iota$ is also invariant under isometries 
of the Riemannian structure and commutes with the action of the Laplace operator $\Delta_{\rho}$ corresponding to $\rho$ on distributions and generalized functions, respectively.

Let $u \in \mathcal{D}^{\prime}(\mathbb{R} \times S)$. Then according to (3.1) the difference between the wave operator $\square_{\lambda}$ and the Laplace operator $\Delta_{\rho}$ acts on $u$ as

$$
\square_{\lambda} u-\Delta_{\rho} u=\frac{-2}{\sqrt{\beta \operatorname{det} h_{t}}} \partial_{t}\left(\sqrt{\frac{\operatorname{det} h_{t}}{\beta}} \partial_{t} u\right):=-2 \Theta u \text {. }
$$

From this equation and the commutation property of the embedding $\iota$ with $\Delta_{\rho}$ we obtain for any $u \in \mathcal{D}^{\prime}(\mathbb{R} \times S)$

$$
\iota\left(\square_{\lambda} u\right)-\square_{\lambda} \iota(u)=\iota\left(\left(\Delta_{\rho}-2 \Theta\right) u\right)-\left(\Delta_{\rho}-2 \Theta\right) \iota(u)=2(\Theta \iota(u)-\iota(\Theta u)) .
$$

Thus, the precise invariance properties of the embedding with respect to the wave operator can be reduced to investigating the corresponding behavior upon interchanging $\iota$ with $\Theta$.

As a first simple, but useful, observation we point out that $\iota$ commutes with $\square_{\lambda}$ in the distributional sense (i.e., in the sense of association in $\mathcal{G}$ ), which follows from the fact that $\square_{\lambda}$ is a differential operator with smooth symbol. This proves the following statement.

Proposition 3.1. For any $u \in \mathcal{D}^{\prime}(\mathbb{R} \times S)$ we have $\iota\left(\square_{\lambda} u\right) \approx \square_{\lambda} \iota(u)$.

We will now show that under additional regularity assumptions on the distribution $u$ a stronger asymptotic property holds.

Theorem 3.2. If $u \in H^{3}(\mathbb{R} \times S)$ is compactly supported then

$$
\left\|\left[T_{\varepsilon}, \square_{\lambda}\right] u\right\|_{L^{2}}=\mathcal{O}\left(\varepsilon^{2}\right) \quad(\varepsilon \rightarrow 0) .
$$

Proof. As in (3.4) we have the basic relation

$$
\left[T_{\varepsilon}, \square_{\lambda}\right] u=2\left[\Theta, T_{\varepsilon}\right] u
$$

Now recalling the Bochner-integral defining the action of $T_{\varepsilon}$ on $L^{2}$-functions we have

$$
\Theta T_{\varepsilon} u-T_{\varepsilon} \Theta u=\frac{1}{2 \pi} \int_{\mathbb{R}} \phi_{c}(s) \frac{1}{\varepsilon} \widehat{F}\left(\frac{s}{\varepsilon}\right)\left(\Theta \cos \left(s \sqrt{-\Delta_{\rho}}\right) u-\cos \left(s \sqrt{-\Delta_{\rho}}\right) \Theta u\right) d s .
$$

Hence everything boils down to deriving asymptotic estimates for the commutator of $\Theta$ with the operator $S(s):=\cos \left(s \sqrt{-\Delta_{\rho}}\right)(s \in \mathbb{R})$. For any $v \in L^{2}(\mathbb{R} \times S)$ the function $w \in \mathcal{C}^{\infty}\left(\mathbb{R}, L^{2}(\mathbb{R} \times S)\right), w(s):=S(s) v(s \in \mathbb{R})$, is the mild solution to the following Cauchy problem on $\mathbb{R} \times \mathbb{R} \times S$ :

$$
\partial_{s}^{2} w-\Delta_{\rho} w=0, \quad w(0)=v, \quad \partial_{s} w(0)=0 .
$$

In the above integral formula the term $\cos \left(s \sqrt{-\Delta_{\rho}}\right) \Theta u$ corresponds to the solution with $v=\Theta u$, whereas the term $\Theta \cos \left(s \sqrt{-\Delta_{\rho}}\right) u$ is just the application of $\Theta$ to the solution $w(s)$ corresponding to $v=u$. Applying $\Theta$ to $(*)$ we obtain

$$
\partial_{s}^{2} \Theta w-\Delta_{\rho} \Theta w=\left[\Theta, \Delta_{\rho}\right] w=: f
$$

and

$$
\left.\Theta w\right|_{s=0}=\Theta(w(0))=\Theta u,\left.\quad \partial_{s} \Theta w\right|_{s=0}=\Theta\left(\partial_{s} w(0)\right)=0
$$


Therefore the Duhamel principle yields

$$
\begin{aligned}
\Theta \cos \left(s \sqrt{-\Delta_{\rho}}\right) u & =\Theta w(s) \\
& =\cos \left(s \sqrt{-\Delta_{\rho}}\right) \Theta u+\int_{0}^{s}(s-r) \operatorname{sinc}\left((s-r) \sqrt{-\Delta_{\rho}}\right) f(r) d r,
\end{aligned}
$$

where $\operatorname{sinc}: \mathbb{R} \rightarrow \mathbb{R}$ is given by $\operatorname{sinc}(z)=\frac{\sin (z)}{z}$. In summary, we obtain

$$
\Theta T_{\varepsilon} u-T_{\varepsilon} \Theta u=\frac{1}{2 \pi} \int_{\mathbb{R}} \phi_{c}(s) \frac{1}{\varepsilon} \widehat{F}\left(\frac{s}{\varepsilon}\right) \int_{0}^{s}(s-r) \operatorname{sinc}\left((s-r) \sqrt{-\Delta_{\rho}}\right) f(r) d r d s,
$$

where

$$
f(r)=\left[\Theta, \Delta_{\rho}\right] w(r)=\left[\Theta, \Delta_{\rho}\right] \cos \left(r \sqrt{-\Delta_{\rho}}\right) u .
$$

Since $\left[\Theta, \Delta_{\rho}\right]$ is of third order and maps $H_{\text {comp }}^{3}$ into $L^{2}$ and $\cos \left(r \sqrt{-\Delta_{\rho}}\right)$ has operator norm 1 on every Sobolev space there exists a constant $C_{1}>0$ such that $\|f(r)\|_{L^{2}} \leq C_{1}\|u\|_{H^{3}}$ for every $r \in \mathbb{R}$. Furthermore, the operator norm of $(s-r) \operatorname{sinc}\left((s-r) \sqrt{-\Delta_{\rho}}\right)$ is bounded by $\sup _{z \in \mathbb{R}}|(s-r) \operatorname{sinc}((s-r) z)|=|s-r|$. Combining these upper bounds we estimate

$$
\begin{aligned}
\| \Theta T_{\varepsilon} u & -T_{\varepsilon} \Theta u\left\|_{L^{2}} \leq \frac{C_{1}}{2 \pi} \int_{\mathbb{R}}\left|\phi_{c}(s)\right| \frac{1}{\varepsilon}\left|\widehat{F}\left(\frac{s}{\varepsilon}\right)\right| \frac{s^{2}}{2} d s \cdot\right\| u \|_{H^{3}} \\
& =\frac{C_{1}}{4 \pi}\|u\|_{H^{3}} \int_{\mathbb{R}}\left|\phi_{c}(\varepsilon \sigma)\left\|\widehat{F}(\sigma) \mid \sigma^{2} d \sigma \cdot \varepsilon^{2} \leq \frac{C_{1}}{4 \pi}\right\| u\left\|_{H^{3}}\right\| \phi_{c}\left\|_{L^{\infty}}\right\| \widehat{F^{\prime \prime}} \|_{L^{1}} \cdot \varepsilon^{2} .\right.
\end{aligned}
$$

Remark 3.3. Applying the reasoning of the proof of Theorem 3.2 to the operators $\partial_{t}$ or $M_{\alpha}$ (multiplication by $\alpha$ ) instead of $\Theta$ gives the following additional asymptotic properties:

(i) If $u \in H^{2}(\mathbb{R} \times S)$ then $\left\|T_{\varepsilon} \partial_{t} u-\partial_{t} T_{\varepsilon} u\right\|_{L^{2}}=\mathcal{O}\left(\varepsilon^{2}\right)(\varepsilon \rightarrow 0)$.

(ii) Let $\alpha \in \mathcal{C}^{\infty}(\mathbb{R} \times S)$ and let $u \in H^{1}(\mathbb{R} \times S)$ have compact support. Then

$$
\left\|T_{\varepsilon}(\alpha u)-\alpha T_{\varepsilon} u\right\|_{L^{2}}=\mathcal{O}\left(\varepsilon^{2}\right) \quad(\varepsilon \rightarrow 0)
$$

We have constructed the regularization operators $\left(T_{\varepsilon}\right)_{\varepsilon \in I}$ and the embedding $\iota$ of distributions on $\mathbb{R} \times S$ based on the Riemannian metric $\rho$ given by 3.2). However, the construction itself does not directly reflect the metric splitting in (3.2) or (3.1) and so far we have not paid special attention to the foliation by the space-like Cauchy hypersurfaces $\{t\} \times S(t \in \mathbb{R})$. However, this foliation becomes essential in case of distributions that allow restriction to these hypersurfaces. The latter is true in particular for distributional solutions to the wave equation $\square_{\lambda} u=f$, where $f \in \mathcal{C}^{\infty}\left(\mathbb{R}, \mathcal{D}^{\prime}(S)\right)$. In these situations we automatically have $u \in \mathcal{C}^{\infty}\left(\mathbb{R}, \mathcal{D}^{\prime}(S)\right)$, since $\operatorname{Char}\left(\square_{\lambda}\right)$ does not contain any elements of the form $(t, x ; \pm 1,0) \in T^{*}(\mathbb{R} \times S)$ (cf. [15], 23.65.5). Thus for every $t \in \mathbb{R}$ the value $u(t)$ is an element of $\mathcal{D}^{\prime}(S)$ and the metric splitting (3.1) provides us with a Riemannian metric $h_{t}$ on $S$, which can be used to regularize or embed $u(t)$ according to our general construction. For every $t \in \mathbb{R}$ let $\left(T_{\varepsilon}^{h_{t}}\right)_{\varepsilon \in I}$ denote the regularization obtained from the Riemann metric $h_{t}$ on $S$ and let $\iota_{h_{t}}$ denote the corresponding embedding $\mathcal{D}^{\prime}(S) \hookrightarrow \mathcal{G}(S)$. The following statements compare these with the global constructions on $\mathbb{R} \times S$.

Theorem 3.4. (i) The embedding $\iota: \mathcal{D}^{\prime}(\mathbb{R} \times S) \hookrightarrow \mathcal{G}(\mathbb{R} \times S)$ respects the metric splitting (3.1) in the weak sense: If $u \in \mathcal{C}^{\infty}\left(\mathbb{R}, \mathcal{D}^{\prime}(S)\right)$ then we have

$$
\forall t \in \mathbb{R}: \iota(u)(t) \approx \iota_{h_{t}}(u(t)) .
$$


(ii) If $u \in \mathcal{C}^{\infty}\left(\mathbb{R}, H^{2}(S)\right)$, then we have for every compact subset $Z \subseteq \mathbb{R}$

$$
\sup _{t \in Z}\left\|\left(T_{\varepsilon} u\right)(t)-T_{\varepsilon}^{h_{t}} u(t)\right\|_{L^{2}(S)}=\mathcal{O}\left(\varepsilon^{2}\right) \quad(\varepsilon \rightarrow 0) .
$$

Proof. (ii) Pick $\chi \in \mathcal{D}(\mathbb{R})$ such that $\chi \equiv 1$ near $Z$ and let $\tilde{u}(t):=\chi(t) u(t)$. It then follows from [13, Prop. 3.7 that $\left(T_{\varepsilon} \tilde{u}-T_{\varepsilon} u\right)_{\varepsilon}$ is negligible on $\operatorname{supp}(\tilde{u}-u)^{c} \supseteq Z$. Thus we may without loss of generality assume that the support of $u$ is bounded in $t$, so $u \in L^{2}(\mathbb{R} \times S)$. This allows us to employ the integral formulae defining $T_{\varepsilon}$ and $T_{\varepsilon}^{h_{t}}$ and obtain

$$
\begin{aligned}
& \left(T_{\varepsilon} u\right)(t)-T_{\varepsilon}^{h_{t}} u(t)= \\
& \frac{1}{2 \pi} \int_{\mathbb{R}} \phi_{c}(s) \frac{1}{\varepsilon} \widehat{F}\left(\frac{s}{\varepsilon}\right)(\underbrace{\left(\cos \left(s \sqrt{-\Delta_{\rho}}\right) u\right)(t)}_{=: w(s, t)}-\underbrace{\cos \left(s \sqrt{-\Delta_{h_{t}}}\right) u(t)}_{=: v(s, t)}) d s,
\end{aligned}
$$

where $v, w \in \mathcal{C}^{\infty}\left(\mathbb{R}^{2}, H^{2}(S)\right)$ solve the following Cauchy problems, respectively:

$$
\begin{array}{rllll}
\left(\partial_{s}^{2}-\Delta_{\rho}\right) w=0 & \left(\text { in } \mathcal{D}^{\prime}(\mathbb{R} \times \mathbb{R} \times S)\right), & \left.w\right|_{s=0}=u, & \left.\partial_{s} w\right|_{s=0}=0, \\
\left(\partial_{s}^{2}-\Delta_{h_{t}}\right) v(., t)=0 & \left(\text { in } \mathcal{D}^{\prime}(\mathbb{R} \times S)\right), & v(0, t)=u(t), & \partial_{s} v(0, t)=0 .
\end{array}
$$

Therefore we have at arbitrary, but fixed $t \in \mathbb{R}$

$$
\partial_{s}^{2} w(., t)-\Delta_{h_{t}} w(., t)=\Delta_{\rho} w(., t)-\Delta_{h_{t}} w(., t)=: f_{t},
$$

where $f_{t} \in \mathcal{C}^{\infty}\left(\mathbb{R}, L^{2}(S)\right.$ ), since $u$ (hence $w$ ) has values in $H^{2}(S)$. We observe that $\psi_{t}:=w(., t)-v(., t)$ satisfies the Cauchy problem

$$
\left(\partial_{s}^{2}-\Delta_{h_{t}}\right) \psi_{t}=f_{t} \quad\left(\text { in } \mathcal{D}^{\prime}(\mathbb{R} \times S)\right), \quad \psi_{t}(0)=0, \quad \partial_{s} \psi_{t}=0,
$$

which implies

$$
\psi_{t}(s)=\int_{0}^{s}(s-r) \operatorname{sinc}\left((s-r) \sqrt{-\Delta_{h_{t}}}\right) f_{t}(r) d r .
$$

Hence we obtain

$$
\left\|\psi_{t}(s)\right\|_{L^{2}(S)} \leq\left|\int_{0}^{s}(s-r)\left\|f_{t}(r)\right\|_{L^{2}(S)} d r\right| \leq \frac{s^{2}}{2} \cdot \sup _{|\sigma| \leq|s|}\left\|f_{t}(\sigma)\right\|_{L^{2}(S)}
$$

and therefore arrive at

$$
\begin{gathered}
\left\|\left(T_{\varepsilon} u\right)(t)-T_{\varepsilon}^{h_{t}} u(t)\right\|_{L^{2}(S)} \leq \frac{1}{2 \pi} \int_{\mathbb{R}}\left|\phi_{c}(s)\right| \frac{1}{\varepsilon}\left|\widehat{F}\left(\frac{s}{\varepsilon}\right)\right|\left\|\psi_{t}(s)\right\|_{L^{2}(S)} d s \\
\leq \frac{\varepsilon^{2}}{4 \pi} \int_{-2 c / \varepsilon}^{2 c / \varepsilon}\left|\phi_{c}(\varepsilon \tau)\left\|\widehat{F}(\tau) \mid \tau^{2} \sup _{|\sigma| \leq \varepsilon|\tau|}\right\| f_{t}(\sigma) \|_{L^{2}(S)} d \tau\right. \\
\leq \frac{\varepsilon^{2}}{4 \pi}\left\|\phi_{c}\right\|_{L^{\infty}} \sup _{|\sigma| \leq 2 c}\left\|f_{t}(\sigma)\right\|_{L^{2}(S)}\left\|\widehat{F^{\prime \prime}}\right\|_{L^{1}}=O\left(\varepsilon^{2}\right) \quad(\varepsilon \rightarrow 0)
\end{gathered}
$$

uniformly when $t$ varies in a compact set.

(i) Since association is checked by action on test functions in $\mathcal{D}(S)$ we may reduce to the case that $\operatorname{supp}(u(t))$ is compact. Then $u(t) \in H^{l}(S)$ for some $l \in \mathbb{Z}$ and since $\left(1-\Delta_{h_{t}}\right)^{ \pm l / 2}$ commutes weakly with $\iota$ and strongly with $\iota_{h_{t}}$ the assertion follows from (ii). 


\section{REFERENCES}

[1] Ch. Bär, N. Ginoux, and F. Pfäffle. Wave equations on Lorentzian manifolds and quantization. ESI Lectures in Mathematics and Physics. European Mathematical Society (EMS), Zürich, 2007.

[2] A.N. Bernal and M. Sánchez. Smoothness of time functions and the metric splitting of globally hyperbolic spacetimes. Commun. Math. Phys., 257(1):43-50, 2005.

[3] A.N. Bernal and M. Sánchez. Globally hyperbolic spacetimes can be defined as 'causal' instead of 'strongly causal'. Classical Quantum Gravity, 24(3):745-749, 2007.

[4] J. Chazarain, A. Piriou. Introduction to the theory of linear partial differential equations. Studies in Mathematics and Its Applications, Vol. 14. North-Holland, 1982.

[5] Y. Choquet-Bruhat. General relativity and the Einstein equations. Oxford Mathematical Monographs. Oxford University Press, Oxford, 2009.

[6] C.J.S. Clarke. The Analysis of Space-Time Singularities, Cambridge University Press, Cambridge, 1993.

[7] C.J.S. Clarke. Singularities: boundaries or internal points?, in Singularities, Black Holes and Cosmic Censorship, Joshi, P. S. and Raychaudhuri, A. K., eds., IUCCA, Bombay, 1996, pp. 2432.

[8] C.J.S. Clarke. Generalized hyperbolicity in singular spacetimes. Class. Quantum Grav. 15 (1998), pp. 975-984.

[9] J.F. Colombeau. New generalized functions and multiplication of distributions. North-Holland, Amsterdam, 1984.

[10] J.F. Colombeau. Elementary introduction to new generalized functions. North-Holland, Amsterdam, 1985.

[11] S. Dave. Geometrical embeddings of distributions into algebras of generalized functions, Math. Nachr., 283 (2010), no. 11, 1575-1588.

[12] J. W. de Roever, M. Damsma. Colombeau algebras on a $C^{\infty}$-manifold. Indag. Math. (N.S.) 2 (1991), no. 3, 341-358.

[13] S. Dave, G. Hörmann, and M. Kunzinger. Optimal regularization processes on complete Riemannian manifolds. 2010. arXiv:1003.3341 [math.FA].

[14] N. Dapić, S. Pilipović, D. Scarpalezos. Microlocal analysis of Colombeau's generalized functions: propagation of singularities. J. Anal. Math. 75 (1998), 51-66.

[15] J. Dieudonné. Treatise on analysis. Vol. VIII, volume 10 of Pure and Applied Mathematics. Academic Press Inc., Boston, MA, 1993.

[16] Garetto, C.. Topological structures in Colombeau algebras: Topological $\widetilde{\mathbb{C}}$-modules and duality theory. Acta Appl. Math. 88, No. 1, 81-123 (2005).

[17] C. Garetto, G. Hörmann. Microlocal analysis of generalized functions: pseudodifferential techniques and propagation of singularities. Proc. Edinb. Math. Soc. (2) 48 (2005), no. 3, 603-629.

[18] R. Geroch. Domain of dependence. Jour. Math. Phys., 11:437 - 449, 1970

[19] J.D.E. Grant, E. Mayerhofer, R. Steinbauer. The wave equation on singular space-times. Comm. Math. Phys. 285 (2009), no. 2, 399-420.

[20] M. Grosser, M. Kunzinger, M. Oberguggenberger, R. Steinbauer. Geometric theory of generalized functions, Kluwer, Dordrecht, 2001.

[21] S. Haller. Microlocal analysis of generalized pullbacks of Colombeau functions. Acta Appl. Math. 105, no. 1, 83-109 (2009).

[22] S.W. Hawking, G.F.R Ellis. The large scale structure of space-time. Cambridge Monographs on Mathematical Physics, No. 1. Cambridge University Press, 1973.

[23] N. Higson, J. Roe. Analytic K-homology. Oxford Mathematical Monographs, Oxford, 2000.

[24] G. Hörmann. Integration and microlocal analysis in Colombeau algebras of generalized functions. J. Math. Anal. Appl. 239 (1999), no. 2, 332-348.

[25] G. Hörmann, M. Kunzinger, R. Steinbauer. Wave equations on non-smooth space-times, this volume.

[26] A. A. Kosinski. Differential manifolds. Pure and Applied Mathematics 138, Academic Press (1993).

[27] M. Kunzinger, R. Steinbauer. Generalized pseudo-Riemannian geometry. Trans. Amer. Math. Soc. 354 (2002), no. 10, 4179-4199.

[28] J. Leray. Hyperbolic Differential Equations, Lecture Notes, IAS, Princeton, 1953. 
[29] E. Minguzzi and M. Sánchez. The causal hierarchy of spacetimes. In Recent developments in pseudo-Riemannian geometry, ESI Lect. Math. Phys., pages 299-358. Eur. Math. Soc., Zürich, 2008.

[30] K. Nomizu and H. Ozeki. The existence of complete Riemannian metrics. Proc. Am. Math. Soc., 12:889-891, 1961.

[31] M. Oberguggenberger. Multiplication of distributions and applications to partial differential equations. Pitman Research Notes in Mathematics 59. Longman, New York, 1992.

[32] B. O'Neill. Semi-Riemannian geometry, volume 103 of Pure and Applied Mathematics. Academic Press, New York, 1983.

[33] R. Steinbauer and J. Vickers, The use of generalized functions and distributions in general relativity, Class. Quantum Grav. 23 (2006), pp. R91-R114.

[34] J. A. Vickers and J. P. Wilson, Generalized hyperbolicity in conical spacetimes, Class. Quantum Grav. 17 (2000), pp. 1333-1260.

Acknowledgment. G.H. thanks the organizers for support by an EPSRC Pathway to Impact Award. We also acknowledge the support of FWF-projects Y237, P20525, and P23714. Finally, we would like to thank the referee for several comments that have led to improvements in the paper.

Faculty of Mathematics, University of Vienna, Nordbergstrasse 15, A 1090 Wien, AUSTRIA

E-mail address: shantanu.dave@univie.ac.at

Faculty of Mathematics, University of Vienna, Nordbergstrasse 15, A 1090 Wien, AUSTRIA

E-mail address: guenther.hoermann@univie.ac.at

Faculty of Mathematics, University of Vienna, Nordbergstrasse 15, A 1090 Wien, AUSTRIA

E-mail address: michael.kunzinger@univie.ac.at 